Publ. RIMS, Kyoto Univ.

12 (1976), 215-227

\title{
Extension of Solutions of Systems of Linear Differential Equations
}

By

Takahiro $\mathrm{KAWAI}^{1)}$

The purpose of this paper is to prove some theorems on the extensibility of hyperfunction solutions and real analytic solutions of systems of linear differential equations with real analytic coefficients.

It is Ehrenpreis [2] that revealed the intimate relations between the algebraic character of "overdetermined" systems of linear differential equations and Hartogs' theorem on the removable singularity of holomorphic functions of several complex variables. In fact, the essential part of his results can be summarized as follows: if a (generalized) function satisfies an "overdetermined" system of linear differential equations with constant coefficients outside a compact set in $\boldsymbol{R}^{n}$, then it must be extended uniquely all over $\boldsymbol{R}^{n}$ as a solution of the same system. Note that the notion of "overdetermined" system is purely algebraic and that the Cauchy-Riemann equation, which holomorphic functions of $n$ complex variables satisfy, is overdetermined if (and only if) $n \geqq 2$. Thus Ehrenpreis [2] has shown that the Hartogs' phenomenon, one of the most interesting features in the theory of holomorphic functions of several complex variables, can be most neatly explained by the general theory of overdetermined systems of linear differential equations. See also Ehrenpreis [3], Palamodov [1], Malgrange [1], Komatsu [1], Kaneko [1], Kawai [3], Bedford-Kawai [1] and references cited there for the further development of the study on the removable singularities of solutions of systems of linear differential equations with constant coefficients. However, the applicability of the methods of these works is strictly confined

Received November 26, 1975.

1) Supported by Miller Institute for Basic Research in Science, University of California. Research Institute for Mathematical Sciences, Kyoto University and Department of Mathematics, University of California, Berkeley 
to the case of the differential equations with constant coefficients. In fact, the results quoted above are more or less dependent on the "Fundamental Principle" of Ehrenpreis [1] for solutions of systems of linear differential equations with constant coefficients and it is impossible to generalize "Fundamental Principle" to the general case of differential equations with variable coefficients as it stands, because of the celebrated counterexample of Lewy [1] against the solvability of linear differential equations. At the same time, in view of the importance of Hartogs' theorem, it is highly desirable to generalize the result of Ehrenpreis [2] to systems of linear differential equations with variable coefficients, i.e. to find out some methods which are independent from "Fundamental Principle" and can be used to investigate the extensibility of solutions of linear differential equations, if possible. The present writer would like to sincerely thank Professor Ehrenpreis for having raised privately the question whether the generalization of this sort is possible or not. At first sight, such a generalization might seem to require many complicated assumptions in view of the phenomenon given by Theorem 2 below, which nullifies the hope of straight forward extension of the results obtained for systems of linear differential equations with constant coefficients. (Cf. Kaneko [1]). However, the recent development in the general theory of systems of (pseudo-) differential equations has enabled us to achieve such a generalization in a desirable and satisfactory manner in the sense that a sufficient condition for the extensibility of hyperfunction solutions is formulated in a purely algebraic manner along the same line as in the case of differential equations with constant coefficients (Theorem 1) and that the results for the extensibility of real analytic solutions are given in terms of algebraic conditions supplemented by natural contact geometric conditions on the characteristic variety of the system involved (Theorems 3,4 and 5). Throughout this paper, it is always assumed that the differential equations involved are linear and with real analytic coefficients and defined on an real analytic manifold. In the course of the proof, we essentially use the theory of boundary value problems for (elliptic) systems of linear differential equations developed by Kashiwara-Kawai [1], [2], [3] and Sato-Kawai-Kashiwara [1] Chapter II and the structure theorem for systems of pseudo-differential equations obtained by Sato- 
Kawai-Kashiwara [1] Chapter III. We use the same notations as in Sato-Kawai-Kashiwara [1] unless otherwise stated. A part of the results in this paper has been announced in Kawai [2].

Firstly we discuss the extensibility of hyperfunction solutions of a system $\mathcal{M}$ of linear differential equations. Clearly Theorem 1 below generalizes a result of Ehrenpreis [3], where $\mathscr{M}$ is assumed to be with constant coefficients and $N$ is assumed to be a linear variety in $\boldsymbol{R}^{n}$. (See p. 346 of Ehrenpreis [3].)

Theorem 1. Let $N$ be a submanifold of a real analytic manifold $M$ of codimension $d \geqq 2$. Assume that $N$ is non-characteristic with respect to a system $M$ of linear differential equations with real analytic coefficients defined on $M$. Then, for any hyperfunction solution $u(x)$ of $\mathscr{M}$ defined on $M-N$, we can find a unique hyperfunction solution $\widetilde{u}(x)$ of $\mathcal{M}$ defined on $M$ so that $\widetilde{u}(x)$ coincides with $u(x)$ on $M-N$.

Remark. Note that the formulation of the non-characteristic property is given in a purely algebraic manner in Sato-Kawai-Kashiwara [1] Chapter II Definition 3.5.4. In our formulation above, the "overdetermined" character of system $\mathcal{M}$ is expressed in terms of the non-characteristic property of higher codimensional submanifold $N$ with respect to $\mathcal{M}$. In fact, Theorem 3.5.6 of Sato-Kawai-Kashiwara [1] Chapter II implies that $\operatorname{Ext}_{\mathscr{P}_{M}}^{1}\left(\mathscr{M}, \mathscr{L}_{M}\right)=0$ if a submanifold $N$ with codimension $d \geqq 2$ of $M$ is non-characteristic with respect to $\mathscr{M}$ because of the trivial fact that $\operatorname{Ext}_{\mathscr{P}_{N}}^{-1}\left(\mathscr{M}_{N}, \mathscr{L}_{N}\right)=0$. Here $\mathscr{L}_{M}\left(\mathscr{L}_{N}\right.$, respectively) denotes the sheaf of pseudo-differential operators defined on (a complexification of) pure imaginary cotangential sphere bundle $\sqrt{-1} S^{*} M\left(\sqrt{-1} S^{*} N\right.$, respectively) and $\mathscr{M}_{N}$ denotes the system induced from $\mathscr{M}$ onto $N$. See Definition 3.5.4. of Sato-Kawai-Kashiwara [1] Chapter II for the precise definition of the notion of the induced system. Note that the induced system $\mathcal{M}_{N}$ is a system of linear differential equations in our case since $\mathcal{M}$ is so.

Proof of Theorem 1. First consider long exact sequence (1) of 
relative cohomology groups associated with $\mathscr{M}$ :

$$
\begin{aligned}
0 & \rightarrow \operatorname{Ext}_{N}{ }^{0}\left(M ; \mathscr{M}, \mathscr{B}_{M}\right) \rightarrow \operatorname{Ext}^{0}\left(M ; \mathscr{M}, \mathscr{B}_{M}\right) \\
& \rightarrow \operatorname{Ext}^{0}\left(M-N ; \mathscr{M}, \mathscr{B}_{M}\right) \rightarrow \operatorname{Ext}_{N}{ }^{1}\left(M ; \mathscr{M}, \mathscr{B}_{M}\right) \\
& \rightarrow \operatorname{Ext}^{1}\left(M ; \mathscr{M}, \mathscr{B}_{M}\right) \rightarrow \cdots .
\end{aligned}
$$

Here $\mathscr{B}_{M}$ denotes sheaf of hyperfunctions on $M$.

Now recall that saying that $u(x)$ is a hyperfunction solution of system $\mathscr{M}$ defined on $M-N(M$, respectively $)$ is equivalent to saying that $u(x)$ is an element in $\operatorname{Ext}^{0}\left(M-N ; \mathscr{M}, \mathscr{B}_{M}\right)\left(\operatorname{Ext}^{0}\left(M ; \mathscr{M}, \mathscr{B}_{M}\right)\right.$, respectively). Therefore, in view of (1), the vanishing of $\operatorname{Ext}_{N}{ }^{1}\left(M ; \mathscr{M}, \mathscr{B}_{M}\right)$ means the extensibility of solution $u(x)$ across $N$ and the vanishing of $\operatorname{Ext}_{N}{ }^{0}\left(M ; \mathscr{M}, \mathscr{B}_{M}\right)$ means the uniqueness of the extension. Thus it suffices to show that $\operatorname{Ext}_{N}{ }^{0}\left(M ; \mathscr{M}, \mathscr{B}_{M}\right)=\operatorname{Ext}_{N}{ }^{1}\left(M ; \mathscr{M}, \mathscr{B}_{M}\right)=0$. On the other hand, Corollary 3.5.8 of Sato-Kawai-Kashiwara [1] Chapter II implies that

$$
\operatorname{Ext}^{j-d}\left(N ; \mathscr{M}_{N}, \mathscr{B}_{N}\right) \underset{\rightarrow}{\rightarrow} \operatorname{Ext}_{N}^{j}\left(M ; \mathscr{M}, \mathscr{B}_{M}\right) \otimes \omega_{N / M}
$$

holds for every $j$, since $N$ is non-characteristic with respect to $\mathscr{M}$ and since $N$ is of codimension $d$ in $M$. Here $\omega_{N / M}=\mathcal{H}_{N}{ }^{d}\left(\boldsymbol{Z}_{M}\right)$. (We refer the reader to Kashiwara-Kawai [3] pp. $4 \sim 5$ for the detailed proof of the above quoted corollary of Sato-Kawai-Kashiwara [1].) Since $\operatorname{Ext}^{k}\left(N ; \mathscr{M}_{N}, \mathscr{B}_{N}\right)$ vanishes for $k<0$ by the definition, (2) implies that $\operatorname{Ext}_{N}{ }^{j}\left(M ; \mathscr{M}, \mathscr{B}_{M}\right)$ vanishes for $j<d$. Since $d \geqq 2$ by the assumption, this implies that $\operatorname{Ext}_{N}{ }^{0}\left(M ; \mathscr{M}, \mathscr{B}_{M}\right)=\operatorname{Ext}_{N}{ }^{1}\left(M ; \mathscr{M}, \mathscr{B}_{M}\right)=0$. This completes the proof of the theorem.

Remark 1. It is obvious from the way of the proof given above that any cohomology class in $\operatorname{Ext}^{j}\left(M-N ; \mathscr{M}, \mathscr{B}_{M}\right)$ can be uniquely extended to a cohomology class in $\operatorname{Ext}^{j}\left(M ; \mathscr{M}, \mathscr{B}_{M}\right)$ if $j<d-1$.

Remark 2. The proof of isomorphism (2) relies essentially on the division theorem of Weierstrass type for (pseudo-) differential operators. (See the proof of Theorem 3.5.6 of Sato-Kawai-Kashiwara [1] Chapter II.) One could bypass the use of the division theorem and prove Theo rem 1 directly by the Serre duality and the existence theorem of Cauchy- 
Kowalevsky. (Cf. Komatsu-Kawai [1]) However, the author believes that the proof employed here is the most straightforward one of the sort.

Secondly we discuss the extensibility of real analytic solutions of a system $\mathscr{M}$ of linear differential equations. One interesting phenomenon in this case is the following:

It may happen that a hyperfunction solution of a system of linear differential equations can be uniquely extended across a submanifold $N$ as a hyperfunction solution but that a real analytic solution of the same system cannot be extended across $N$ as a real analytic solutions. (Theorem 2.)

It is worth while emphasizing that such a phenomenon can never be observed in the case of systems of linear differential equations with constant coefficients. See the remark after Theorem 2 .

We also give some affirmative results for systems with variable coefficients. (Theorems 3 and 4 below.)

Theorem 2. Assume that a submanifold $N$ of $M$ and a system $M$ of linear differential equations defined on $M$ satisfy conditions given in Theorem 1. Assume that there exists a point $x_{0}$ in $N$ and a non-zero cotangent vector $\eta_{0}$ at $x_{0}$ such that the generalized Levi form associated with the characteristic variety $V$ of $\mathscr{M}$ is non-degenerate and has signature $(r, 0)$ at $\left(x_{0}, \sqrt{-1} \eta_{0}\right) \in \sqrt{-1} S^{*} M$. Here $r$ denotes the codimensionality of $V$ in a complexification of $\sqrt{-1} S^{*} M$. Assume that $\mathscr{M}$ is regular in the sense that $\operatorname{Ext}\left(\mathscr{M}, \mathscr{D}_{M}\right)=0$ for $j \neq r$. Here $\mathscr{D}_{M}$ denotes sheaf of linear differential operators defined on $M$. Assume further that codim $N=r$. Then there exist a neighborhood $U$ of $x_{0}$ and a real analytic solution $u(x)$ of $\mathcal{M}$ defined on $U-\left\{x_{0}\right\}$ such that we can never find a real analytic solution $\widetilde{u}(x)$ of $\mathcal{M}$ defined on $U$ which coincides with $u(x)$ on $U-\left\{x_{0}\right\}$, though $u(x)$ can be extended as a hyperfunction solution of $\mathcal{M}$ defined on $U$.

Proof. Remark in p. 417 of Sato-Kawai-Kashiwara [1] claims that $\mathscr{M}_{N}$ is a locally projective left $\mathscr{D}_{N}$-Module. Since Theorem 2.3.4 of Kashiwara [1] asserts that $\underset{l}{\lim } \operatorname{Ext}^{1}\left(U_{l} ; \mathscr{M}, \mathcal{A}_{M}\right)$ is isomorphic to 
$\underset{l}{\lim _{l}} \operatorname{Ext}^{1}\left(U_{l} \cap N ; \mathscr{M}_{N}, \mathscr{A}_{N}\right)$ for a fundamental system of neighborhoods $\left\{U_{l}\right\}_{l=1}^{\infty}$ of $x_{0}$ in $M$, this implies that $\underset{l}{\lim } \operatorname{Ext}^{1}\left(U_{l} ; \mathcal{M}, \mathcal{A}_{M}\right)$ vanishes. Here $\mathcal{A}_{M}$ denotes sheaf of real analytic functions defined on $M$. On the other hand we have the following long exact sequence of relative cohomology groups:

$$
\begin{aligned}
& 0 \rightarrow \underset{l}{\lim } \operatorname{Ext}_{\left\{x_{0}\right\}}^{0}\left(U_{l} ; \mathscr{M}, \mathcal{A}_{M}\right) \rightarrow \underset{l}{\lim } \operatorname{Ext}^{0}\left(U_{l} ; \mathscr{M}, \mathcal{A}_{M}\right) \\
& \rightarrow \underset{l}{\lim } \operatorname{Ext}^{0}\left(U_{l}-\left\{x_{0}\right\} ; \mathscr{M}, \mathcal{A}_{M}\right) \rightarrow \underset{l}{\rightarrow} \lim _{\left\{x_{0}\right\}}^{1}\left(U_{l} ; \mathcal{M}, \mathcal{A}_{M}\right) \\
& \rightarrow \underset{l}{\lim } \operatorname{Ext}^{1}\left(U_{l} ; \mathscr{M}, \mathscr{A}_{M}\right) \rightarrow \cdots .
\end{aligned}
$$

Hence it suffices to show that $\underset{l}{\lim } \operatorname{Ext}_{\left\{x_{0}\right\}}^{1}\left(U_{l} ; \mathscr{M}, \mathcal{A}_{M}\right) \neq 0$. In fact, the fact that $\underset{l}{\longrightarrow} \operatorname{Ext}_{\left\{x_{0}\right\}}^{1}\left(U_{l} ; \mathscr{M}, \mathcal{A}_{M}\right) \neq 0$ combined with the vanishing of $\underset{l}{\lim } \operatorname{Ext}^{1}\left(U_{l} ; \mathscr{M}, \mathcal{A}_{M}\right)$ implies that there exists a real analytic solution of $\overrightarrow{\mathscr{M}}$ defined on $U_{l_{0}}-\left\{x_{0}\right\}$ for some $l_{0}$ which can never be a restriction of a real analytic solution of $\mathcal{M}$ defined in a neighborhood of $x_{0}$.

On the other hand, under the assumptions of Theorem 1, $\underset{l}{\lim } \operatorname{Ext}_{\left\{x_{0}\right\}}^{1}\left(U_{l} ; \mathscr{M}, \mathcal{A}_{M}\right)$ never vanishes if $\underset{l}{\lim } \operatorname{Ext}_{\left\{x_{0}\right\}}^{0}\left(U_{l} ; \mathscr{M}, \mathscr{B}_{M} / \mathcal{A}_{M}\right) \neq 0$, because the following long exact sequence of relative cohomology groups exists.

$$
\begin{aligned}
0 & \rightarrow \underset{l}{\lim } \operatorname{Ext}_{\left\{x_{0}\right\}}^{0}\left(U_{l} ; \mathscr{M}, \mathscr{A}_{M}\right) \rightarrow \underset{l}{\lim } \operatorname{Ext}_{\left\{x_{0}\right\}}^{0}\left(U_{l} ; \mathscr{M}, \mathscr{B}_{M}\right) \\
& \rightarrow \underset{l}{\lim } \operatorname{Ext}_{\left\{x_{0}\right\}}^{0}\left(U_{l} ; \mathscr{M}, \mathscr{B}_{M} / \mathcal{A}_{M}\right) \rightarrow \underset{l}{\lim } \operatorname{Ext}_{\left\{x_{0}\right\}}^{1}\left(U_{l} ; \mathscr{M}, \mathcal{A}_{M}\right) \\
& \rightarrow \underset{l}{\lim } \operatorname{Ext}_{\left\{x_{0}\right\}}^{1}\left(U_{l} ; \mathscr{M}, \mathscr{B}_{M}\right) \rightarrow \cdots .
\end{aligned}
$$

In fact, under the assumptions of Theorem 1, it is clear that $\operatorname{Ext}_{\left\{x_{0}\right\}}^{0}\left(U_{l} ; \mathscr{M}, \mathscr{B}_{M}\right)=0$. Hence the vanishing of $\underset{l}{\lim } \operatorname{Ext}_{\left\{x_{0}\right\}}^{1}\left(U_{l} ; \mathscr{M}, \mathcal{A}_{M}\right)$ implies that of $\underset{l}{\lim } \operatorname{Ext}_{\left\{x_{0}\right\}}^{0}\left(U_{l} ; \mathcal{M}, \mathscr{B}_{M} / \mathcal{A}_{M}\right)$. Further, it is easy to see that $\operatorname{Ext}_{\left\{x_{0}\right\}}^{1}\left(U_{l} ; \mathscr{M}, \mathcal{A}_{M}\right)$ is isomorphic to $\operatorname{Ext}_{\left\{x_{0}\right\}}^{0}\left(U_{l} ; \mathscr{M}, \mathscr{B}_{M} / \mathscr{A}_{M}\right)$ in our case, though we need not use this precise statement at this stage. As a matter of fact, the way of the proof of Theorem 1 shows that $\operatorname{Ext}_{K}{ }^{0}\left(M ; \mathscr{M}, \mathscr{B}_{M}\right)$ $=\operatorname{Ext}_{K}{ }^{1}\left(M ; \mathscr{M}, \mathscr{B}_{M}\right)=0$ for any closed set $K$ in $M$.

Therefore it suffices to show that $\underset{l}{\lim } \operatorname{Ext}_{\left\{x_{0}\right\}}^{0}\left(U_{l} ; \mathcal{M}, \mathscr{B}_{M} / \mathcal{A}_{M}\right) \neq 0$. To prove this fact, we resort to the fundamental structure theorem for 
overdetermined systems of pseudo-differential equations. In fact, Corollary 2.4.3 of Sato-Kawai-Kashiwara [1] Chapter III shows that $\operatorname{Ext}_{\left\{\left(x_{0}, \sqrt{-1} \eta_{0}\right)\right\}}^{0}\left(\sqrt{-1} S^{*} U_{l} ; \mathscr{L}_{M_{\pi^{-1}} \mathscr{D}_{\mathcal{K}}} \underset{M}{M} \mathscr{C}_{M}\right) \neq 0$ under the assumptions of the theorem. Here $\mathscr{C}_{M}$ denotes sheaf of microfunctions defined on $\sqrt{-1} S^{*} M$ and $\pi$ denotes the canonical projection from $\sqrt{-1} S^{*} M$ onto $M$. Since $\pi_{*} \mathscr{C}_{M}$ is isomorphic to $\mathscr{B}_{M} / \mathcal{A}_{M}$, this implies that $\underset{l}{\lim } \operatorname{Ext}_{\left\{x_{0}\right\}}^{0}\left(U_{l} ; \mathscr{M}, \mathscr{B}_{N} / \mathcal{A}_{M}\right)$ $\neq 0$. This completes the proof of the theorem.

Remark. If $\mathcal{M}$ is a system of linear differential equations with constant coefficients, $\operatorname{Ext}_{\left\{x_{0}\right\}}^{0}\left(M ; \mathscr{M}, \mathscr{B}_{M} / \mathcal{A}_{M}\right)=0$ holds under the assumption that $\mathcal{M}$ is a determined system. (Kawai [1] Theorem 5.2.1) This fact clearly implies that $\operatorname{Ext}_{\left\{x_{0}\right\}}^{1}\left(M ; \mathscr{M}, \mathcal{A}_{M}\right)$ must necessarily vanish under the assumptions of Theorem 1. This is the reason why the phenomenon observed in Theorem 2 has never been found for systems of linear differential equations with constant coefficients.

In order to prove affirmative results for the extensibility of real analytic solutions, we recall the notion of virtual bicharacteristic manifold of a system $\mathscr{M}$ of pseudo-differential equations which satisfies following conditions $(5) \sim(7)$. There $V$ denotes the characteristic variety of $\mathscr{M}$ considered in a complexification of $\sqrt{-1} S^{*} M$ and $\bar{V}$ denotes the variety complex conjugate to $V$.

(5) $\quad V$ is non-singular and $T_{x^{*}}(V) \cap T_{x^{*}}(\bar{V})=T_{x^{*}}(V \cap \bar{V})$ holds for any $x^{*}=(x, \sqrt{-1} \eta)$ in $V \cap \sqrt{-1} S^{*} M$.

(6) $\quad V \cap \bar{V}$ is regular in the sense of Definition 3.3.1 of Sato-Kawai-Kashiwara [1] Chapter II, i.e. $\left.\omega\right|_{V \cap \bar{V}}$ never vanishes on $V \cap \bar{V}$, where $\omega$ denotes the fundamental 1 -form of $\sqrt{-1} S^{*} M$.

(7) The generalized Levi form $V$ is of constant signature $(p, q)$ in a neighborhood of any point $\left(x_{0}, \sqrt{-1} \eta_{0}\right) \in V \cap \sqrt{-1} S^{*} M$.

It is known that under assumptions $(5) \sim(7)$ we can find complex manifold $V_{j}(j=1,2,3)$ which satisfy the following:

(8) $\quad V=V_{1} \cap V_{2} \cap V_{3}$

(9) $\quad V_{1}$ is a complexification of a real involutory manifold.

(10) $V_{2}$ and $\bar{V}_{2}$ intersect transversally at any point of $V_{2} \cap \sqrt{-1} S^{*} M$.

(11) $V_{2} \cap \sqrt{-1} S^{*} M$ is involutory and regular in $\sqrt{-1} S^{*} M$. 
(12) The generalized Levi form of $V_{3}$ is of signature $(p, q)$ and codim $V_{3}=p+q$.

(See p. 523 of Sato-Kawai-Kashiwara [1].)

Now we give the definition of virtual bicharacteristic manifold of $\mathscr{M}$. (Cf. Definition 2.2.7 of Sato-Kawai-Kashiwara [1] Chapter III.)

Definition. The virtual bicharacteristic manifold $\Lambda_{\left(x_{0}, \sqrt{-1} \eta_{0}\right)}$ of $\mathscr{M}$ through $\left(x_{0}, \sqrt{-1} \eta_{0}\right) \in V \cap \sqrt{-1} S^{*} M$ is the real $\left(\left(\operatorname{codim} V_{1}+2 \operatorname{codim} V_{2}\right)\right.$ -dimensional) bicharacteristic manifold through $\left(x_{0}, \sqrt{-1} \eta_{0}\right)$ of real involutory manifold $V_{1} \cap V_{2} \cap \sqrt{-1} S^{*} M$.

A theorem which makes a counterpart to Theorem 2 is now given.

Theorem 3. Let $\mathcal{M}$ be a system of linear differential equations defined on $M$. Let $N$ be a submanifold of $M$ of codimension $d \geqq 2$. Assume that $N$ is non-characteristic with respect to $\mathcal{M}$. Assume that $\mathcal{M}$ is a regular system which satisfies conditions (5) $(7)$ for any point in $\left(x_{0}, \sqrt{-1} \eta_{0}\right){ }^{2)}$ Assume that $\mathcal{M}$ satisfies either one of the following two conditions (13) and (14):

(13) $q \geqq 1$

(14) For any point $(x, \sqrt{-1} \eta)$ in $V \cap \sqrt{-1} S^{*} M \times x$ there exists a virtual bicharacteristic manifold $\Lambda_{(x, \sqrt{-1} \eta)}$ of $M$ which passes through $(x, \sqrt{-1} \eta)$ and contains a point $(\widetilde{x}, \sqrt{-1} \tilde{\eta})$ with $\widetilde{x} \notin N$.

Then, under these assumptions, for any real analytic solution $u(x)$ of $M$ defined of $M-N$, we can find a unique real analytic solution $\widetilde{u}(x)$ of $\mathcal{M}$ defined on $M$ so that $\widetilde{u}(x)$ coincides with $u(x)$ on $M-N$.

Proof. In view of the following long exact sequence $\left(3^{\prime}\right)$, it suffices to show that $\operatorname{Ext}_{N}{ }^{1}\left(M ; \mathscr{M}, \mathcal{A}_{M}\right)=0$ holds.

$$
\begin{aligned}
0 & \rightarrow \operatorname{Ext}_{N}{ }^{0}\left(M ; \mathscr{M}, \mathscr{A}_{M}\right) \rightarrow \operatorname{Ext}^{0}\left(M ; \mathscr{M}, \mathscr{A}_{M}\right) \\
& \rightarrow \operatorname{Ext}^{0}\left(M-N ; \mathscr{M}, \mathcal{A}_{M}\right) \rightarrow \operatorname{Ext}_{N}{ }^{1}\left(M ; \mathscr{M}, \mathscr{A}_{M}\right) \\
& \rightarrow \operatorname{Ext}^{1}\left(M ; \mathscr{M}, \mathscr{A}_{M}\right) \rightarrow \cdots .
\end{aligned}
$$

2) $(p, q)$ may be dependent on the connected component of $V \cap \sqrt{-1 S^{*}} M$. 
Further, the following exact sequence $\left(4^{\prime}\right)$ combined with Theorem 1 implies that $\operatorname{Ext}_{N}{ }^{1}\left(M ; \mathscr{M}, \mathcal{A}_{M}\right)$ is isomorphic to $\operatorname{Ext}_{N}{ }^{0}\left(M ; \mathcal{M}, \mathscr{B}_{M} / \mathcal{A}_{M}\right)$.

$$
\begin{aligned}
0 & \rightarrow \operatorname{Ext}_{N}{ }^{0}\left(M ; \mathscr{M}, \mathscr{A}_{M}\right) \rightarrow \operatorname{Ext}_{N}{ }^{0}\left(M ; \mathscr{M}, \mathscr{B}_{M}\right) \\
& \rightarrow \operatorname{Ext}_{N}{ }^{0}\left(M ; \mathscr{M}, \mathscr{B}_{M} / \mathcal{A}_{M}\right) \rightarrow \operatorname{Ext}_{N}{ }^{1}\left(M ; \mathscr{M}, \mathscr{A}_{M}\right) \\
& \rightarrow \operatorname{Ext}_{N}{ }^{1}\left(M ; \mathscr{M}, \mathscr{B}_{M}\right) \rightarrow \cdots
\end{aligned}
$$

Thus the problem has been reduced to the proof of the vanishing of $\operatorname{Ext}_{N}{ }^{0}\left(M ; \mathscr{M}, \mathscr{B}_{M} / \mathcal{A}_{M}\right)$.

First consider the case where (13) is satisfied. Assumption (13) implies that any hyperfunction solution $u(x)$ of $\mathscr{M}$ is real analytic because of Corollary 2.4.2 of Sato-Kawai-Kashiwara [1] Chapter III. Thus (13) trivially implies $\operatorname{Ext}_{N}{ }^{0}\left(M ; \mathscr{M}, \mathscr{B}_{M} / \mathcal{A}_{M}\right)=0$.

Nextly assume that (14) holds. Since singularity of hyperfunction solutions of $\mathscr{M}$ propagates along virtual bicharacteristics in the sense of Sato-Kawai-Kashiwara [1] Chapter III Corollary 2.4.2, there is no hyperfunction solution of $\mathscr{M}$ which is singular only along $N$. This means that $\operatorname{Ext}_{N}{ }^{0}\left(M ; \mathscr{M}, \mathscr{B}_{M} / \mathscr{A}_{M}\right)=0$.

This completes the proof of the theorem.

Remark 1. As is clear from the way of the argument above, regularity condition on the characteristic variety $V$ of $\mathcal{M}$ can be weakened. For example, if the generalized Levi form of $V$ has at least one negative eigenvalues, then the conclusion of the theorem holds without any regularity conditions on $V$ or $\mathscr{M}$. This result follows from Theorem 2.3.10 of Sato-Kawai-Kashiwara [1] Chapter III. In Theorem 3 we assumed the regularity of $V$ so that we can make use of the notion of virtual bicharacteristic manifold. It seems to the author that the use of virtual bicharacteristic manifolds at this stage has its own interest related to the remark after Theorem 2, since condition (13) is never satisfied by a system with constant coefficients.

Remark 2. It is obvious from the way of the proof that any cohomology class in $\operatorname{Ext}^{j}\left(M-N ; \mathcal{M}, \mathcal{A}_{M}\right)$ can be uniquely extended to a cohomology class in $\operatorname{Ext}^{j}\left(M ; \mathscr{M}, \mathscr{A}_{M}\right)$ under the assumption of the regularity 
of $\mathcal{M}$ and the same assumption on $N$ as in Theorem 3,

either

if $j<\min (d-1, q)$

or

if $j<d-1$ and condition (14) is satisfied.

Theorem 4. Let $\mathcal{L}$ be a system of linear differential equations defined on a real analytic manifold $L$. Let $Z$ be a closed subset of $L$. Let $\varphi(x)$ be a real valued real analytic function defined on $L$ such that $\operatorname{grad}_{x} \varphi(x)$ never vanishes if $\varphi(x) \geqq 0$. Assume that hypersurface $S_{t}=\{x \in L ; \varphi(x)=t\}$ is non-characteristic with respect to $\mathcal{L}$ for any $t \geqq 0$. Assume that $S_{t} \cap Z$ is compact for any $t$ and that $S_{t} \cap Z$ is void for $t<0$. Assume that system $\mathcal{L}_{t}$ of linear differential equations induced from $\mathcal{L}$ to $S_{t}$ satisfies conditions $(5) \sim(7)$ when we consider $S_{t}$ as $M$ and $\mathcal{L}_{t}$ as $\mathcal{M}$. Assume that $\mathcal{L}_{t}$ satisfies either one of the following two conditions:

$\left(13^{\prime}\right) \quad q \geqq 1$.

$\left(14^{\prime}\right)$ For any point $\left(x^{\prime}, \sqrt{-1} \eta^{\prime}\right)$ in $V_{t} \cap \sqrt{-1} S^{*}\left(S_{t}\right) \widehat{S}_{t}\left(S_{t} \cap Z\right)$ there exists a virtual bicharacteristic manifold $\Lambda_{\left(x^{\prime}, \sqrt{-1} \eta^{\prime}\right)}$ of $\mathcal{L}_{t}$ which passes through $\left(x^{\prime}, \sqrt{-1} \eta^{\prime}\right)$ and contains a point $\left(\widetilde{x}^{\prime}, \sqrt{-1} \tilde{\eta}^{\prime}\right)$ with $\tilde{x}^{\prime} \in S_{t}-\left(S_{t} \cap Z\right)$. Here $V_{t}$ denotes the characteristic variety of $\mathcal{L}_{t}$.

Then any real analytic solution $u(x)$ of $\mathcal{L}$ defined on $L-Z$ can be extended over $L$ as a real analytic solution of $\mathcal{L}$.

Proof. Define $\Omega_{t}$ by $\{x \in L ; \varphi(x)<t\}$, and define $t_{0}$ by $\sup \{t$; there exists a real analytic solution $\widetilde{u}(x)$ of $\mathcal{L}$ which is defined on $\Omega_{t}$ and coincides with $u(x)$ in $L-Z\}$. It suffices to show that $t_{0}=\infty$. Assume that it were not the case. Then it is clear that $u(x)$ is defined on $\Omega_{t_{0}}$ and satisfies $\mathcal{L}$ there. Since $S_{t_{0}}$ is non-characteristic with respect to $\mathcal{L}$, Corollary 3.5.8 of Sato-Kawai-Kashiwara [1] Chapter II implies the existence of the following isomorphism:

$$
\operatorname{Ext}^{0}\left(S_{t} ; \mathcal{L}_{t}, \mathscr{B}_{s_{t}}\right) \stackrel{\stackrel{s}{\rightarrow}}{\rightarrow} \operatorname{Ext}_{S_{t}}^{1}\left(L ; \mathcal{L}, \mathscr{B}_{L}\right) \otimes \omega_{s_{t} / L}
$$

In view of the long exact sequence 


$$
\begin{aligned}
0 & \rightarrow \operatorname{Ext}_{S_{t_{0}}}^{0}\left(L ; \mathcal{L}, \mathscr{B}_{L}\right) \\
& \rightarrow \operatorname{Ext}^{0}\left(L ; \mathcal{L}, \mathscr{B}_{L}\right) \rightarrow \operatorname{Ext}^{0}\left(L-S_{t_{0}} ; \mathcal{L}, \mathscr{B}_{L}\right) \\
& \stackrel{\delta}{\rightarrow} \operatorname{Ext}_{s_{t_{0}}}^{1}\left(L ; \mathcal{L}, \mathscr{B}_{L}\right) \rightarrow \cdots,
\end{aligned}
$$

any hyperfunction solution of $\mathcal{L}$ defined on $L-S_{t_{0}}$ defines a cohomology class in $\operatorname{Ext}_{\mathcal{S}_{t_{0}}}^{1}\left(L ; \mathcal{L}, \mathscr{B}_{L}\right)$, hence, applied by $c^{-1}$, it defines a cohomology class in $\operatorname{Ext}^{0}\left(S_{t_{0}} ; \mathcal{L}_{t_{0}}, \mathscr{B}_{s_{t_{0}}}\right)$. Now define $\widetilde{u}(x)$ by $u(x)$ on $\Omega_{t_{0}}$ and by 0 on $\left\{x \in L ; \varphi(x)>t_{0}\right\}$. Clearly $\widetilde{u}(x)$ is a real analytic solution of $\mathcal{L}$ defined on $L-S_{t_{0}}$. Therefore $\iota^{-1} \delta(\widetilde{u}(x))$ defines a hyperfunction solution $v\left(x^{\prime}\right)$ of $\mathcal{L}_{t_{0}}$ defined on $S_{t_{0}}$. This is nothing but the boundary value of $u(x)$. It is then obvious that $v\left(x^{\prime}\right)$ is real analytic near $x_{0}^{\prime} \in S_{t_{0}}$ if $u(x)$ is real analytic in a neighborhood of $x_{0}{ }^{\prime}$ in $L$. Therefore $v\left(x^{\prime}\right)$ is a hyperfunction solution of $\mathcal{L}_{t_{0}}$ which is real analytic in $S_{t_{0}}-\left(S_{t_{0}} \cap Z\right)$. Then, by the same argument used in the proof of Theorem 3 , we conclude that $v\left(x^{\prime}\right)$ is real analytic on $S_{t_{0}}$. Thus the boundary value of $u(x)$ on $S_{t_{0}}$ is seen to be real analytic. Then by applying the Cauchy-Kowalevsky theorem for systems of linear differential equations, we can find a real analytic solution $\widetilde{\widetilde{u}}(x)$ of $\mathcal{L}$ defined in a neighborhood of $S_{t_{0}}$ so that it coincides with $u(x)$ on $\Omega_{t_{0}}$. See Kashiwara [1] Theorem 2.3.1, for example, as for the Cauchy-Kowalevsky theorem for systems. Since $S_{t_{0}} \cap Z$ is compact by the assumption, the existence of $\widetilde{\widetilde{u}}(x)$ implies that $u(x)$ can be extended to $\Omega_{t_{0}+\varepsilon}$ for some $\varepsilon>0$ as a real analytic solution of $\mathcal{L}$. This contradicts the definition of $t_{0}$. Therefore $t_{0}$ should be infinite.

This completes the proof of the theorem.

Remark. If we additionally assume that $\mathcal{L}$ is an elliptic system, we can obtain the same result even when we replace the induced system $\mathcal{L}_{t}$ by its direct summand $\left(\mathcal{L}_{t}\right)_{\text {- }}$ as follow: $:^{3)}$

Denote by $L^{\boldsymbol{C}}\left(\left(S_{t}\right)^{\boldsymbol{C}}\right.$, respectively) a complexification of $L\left(S_{t}\right.$, respectively). Define $\left(G_{t}\right)$ - by $\left\{(z, \zeta) \in S_{S_{t}}^{*} L^{c} ; z \in S_{t}, \operatorname{Re} \zeta=-\operatorname{grad}_{z} \varphi(z)\right\}$. Here $S_{S_{t}}^{*} L^{\boldsymbol{C}}$ denotes the conormal bundle supported by $S_{t}$. Denote by

8) As is clear from the definition below, system $\left(\mathcal{L}_{t}\right)_{-}$is not necessarily a system of linear differential equations, but, in general, a system of pseudo-differential equations. 
$\rho$ the canonical projection from $S_{\boldsymbol{S}_{t}}^{*} L^{\boldsymbol{C}}-S_{\left(S_{t}\right)}^{*} \boldsymbol{C} L^{\boldsymbol{C}}$ to $S_{\boldsymbol{S}_{t}}^{*}\left(S_{t}\right)^{\boldsymbol{C}}$. Then ellip-

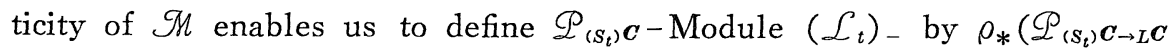

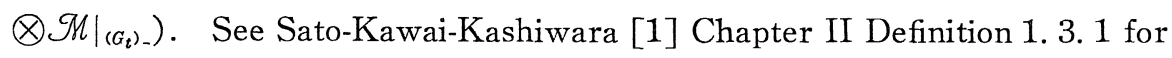
the definition of $\mathscr{L}_{\left(\boldsymbol{S}_{t}\right)} \boldsymbol{C}_{\rightarrow L} \boldsymbol{C}$

In this case, we can use following isomorphism (15) instead of $\left(2^{\prime}\right)$ :

$$
\operatorname{Ext}_{\partial \Omega_{t_{0}}}^{1}\left(\bar{\Omega}_{t_{0}} ; \mathcal{L}, \mathscr{B}_{L}\right) \otimes \omega_{S_{t_{0}} / L} \underset{\rightarrow}{ } \operatorname{Ext}^{0}\left(\sqrt{-1} S^{*}\left(S_{t_{0}}\right) ;\left(\mathcal{L}_{t_{0}}\right)_{-}, \mathscr{C}_{S_{t_{0}}}\right) \text {. }
$$

Isomorphism (15) immediately follows from Theorem 1 of KashiwaraKawai [1]. Further we use following long exact sequence (16) instead of $\left(1^{\prime}\right)$

$$
\begin{aligned}
0 & \rightarrow \operatorname{Ext}_{\partial \Omega_{t_{0}}}^{0}\left(\bar{\Omega}_{t_{0}} ; \mathcal{L}, \mathscr{B}_{L}\right) \rightarrow \operatorname{Ext}^{0}\left(\bar{\Omega}_{t_{0}} ; \mathcal{L}, \mathscr{B}_{L}\right) \\
& \rightarrow \operatorname{Ext}^{0}\left(\Omega_{t_{0}}, \mathcal{L}, \mathscr{B}_{L}\right) \stackrel{\delta}{\rightarrow} \operatorname{Ext}_{\partial \Omega_{t_{0}}}^{1}\left(\bar{\Omega}_{t_{0}} ; \mathcal{L}, \mathscr{B}_{L}\right) \\
& \rightarrow \cdots
\end{aligned}
$$

Then the rest of the argument in the proof of Theorem 4 goes well without any essential changes. Thus we have the following theorem.

Theorem 5. Let $\mathcal{L}, L, Z$ and $\varphi(x)$ be the same as in Theorem 4. Assume further that $\mathcal{L}$ is an elliptic system of linear differential equations. Assume that $\left(\mathcal{L}_{t}\right)$ - satisfies conditions (5), (6), (7) and either $\left(13^{\prime}\right)$ or $\left(14^{\prime}\right)$. Then any (hyperfunction) solution $u(x)$ of $\mathcal{L}$ defined on $L-Z$ can be extended over $L$ as a (hyperfunction) solution of $\mathcal{L}$.

Remark. When $\mathcal{L}$ is a single linear differential equation, Kaneko [2] has recently given interesting results on the extension of real analytic solutions and hyperfunction solutions from the view point of singularity spectrum of boundary values of solutions. In view of Theorem 5 it would be an interesting problem to extend his results to overdetermined systems which are not necessarily elliptic. As a matter of fact, the essential point in the proof of Theorem 5 is the fact that we can easily "microlocalize" the argument on the boundary of $\Omega_{t}$ if $\mathcal{L}$ is elliptic. Presumably the results of Kashiwara-Kawai [4] would be useful for this purpose. 


\section{References}

Bedford, E. and Kawai, T.: [1], Local extension of solutions of systems of linear differential equations with constant coefficients, to appear.

Ehrenpreis, L.: [1], The fundamental principle for linear constant coefficients partial differential equations, Proc. Intern. Symp. Linear Spaces, Jerusalem (1960), pp. $161-174$.

: [2], A new proof and an extension of Hartog's theorem, Bull. Amer. Math. Soc., 67 (1961), 507-509.

: [3], Fourier Analysis in Several Complex Variables, Wiley-Interscience, New York-London-Sydney-Toronto, 1970.

Kaneko, A.: [1], On continuation of regular solutions of partial differential equations to compact convex sets. II, J. Fac. Sci. Univ. Tokyo, 18 (1972), 415-433.

_ : [2], Singular spectrum of boundary values of solutions of partial differential equations with real analytic coefficients, to appear.

Kashiwara, M.: [1], Algebraic study on systems of partial differential equations, Master's thesis presented to Univ. Tokyo (1971). (In Japanese)

and Kawai, T.: [1], On the boundary value problem for elliptic system of linear differential equations. I, Proc. Japan Acad., 48 (1972), 712-715.

: [2], Ibid. II. Ibid., 49 (1973), 164-168.

: [3], Theory of elliptic boundary value problems and its applications. Sûrikaisekikenkyûsho Kôkyûroku, No. 238, Res. Inst. Math. Sci. Kyoto Univ. Kyoto, 1975, 1-59. (In Japanese)

: [4], Micro-hyperbolic pesudo-differential operators I, J. Math. Soc. Japan, 27 (1975), 359-404.

Kawai, T.: [1], On the theory of Fourier hyperfunctions and its applications to partial differential equations with constant coefficients, J. Fac. Sci. Univ. Tokyo, 17 (1970), 467-517.

: [2], Removable singularities of solutions of systems of linear differential equations, Bull. Amer. Math. Soc., 81 (1975), 461-463.

: [3], Extension of hyperfunction solutions of linear differential equations with constant coefficients, to appear in Proc. Amer. Math. Soc.

Komatsu, H.: [1], Relative cohomology of sheaves of solutions of differential equations, Lecture Notes in Math. No. 287, Springer, Berlin-New York-Heidelberg, 1973, pp. 192-261.

- and Kawai, T.: [1], Boundary values of hyperfunction solutions of linear partial differential equations, Publ. RIMS. Kyoto Univ., 7 (1971), 95-104.

Lewy, H.: [1], An example of a smooth linear partial differential equation without solutions, Ann. of Math., 66 (1975), 155-158.

Malgrange, B.: [1], Systèmes différentiels à coefficients constants, Sém. Bourbaki, 15 (1962), Exposé 246.

Palamodov, V. P.: [1], Linear Differential Operators with Constant Coefficients, Nauka, Moscow, 1967. (In Russian. Translated into English (Springer) and into Japanese (Yoshioka))

Sato, M., Kawai, T. and Kashiwara, M.: [1], Microfunctions and pseudo-differential equations, Lecture Notes in Math. No. 287, Springer, Berlin-New York-Heidelberg, 1973. pp. 265-529. 
